\section{La Révolution française}

Cahiers de l'Institut d'histoire de la Révolution française

5 | 2013

Le républicanisme anglais dans la France des

Lumières et de la Révolution

\title{
Le républicanisme anglais dans la France des Lumières et de la Révolution : mesure d'une présence
}

François Quastana et Pierre Serna

\section{(2) OpenEdition}

\section{Journals}

Édition électronique

URL : http://journals.openedition.org//rf/984

DOI : $10.4000 /$ Irf.984

ISSN : 2105-2557

Éditeur

IHMC - Institut d'histoire moderne et contemporaine (UMR 8066)

Référence électronique

François Quastana et Pierre Serna, « Le républicanisme anglais dans la France des Lumières et de la Révolution : mesure d'une présence », La Révolution française [En ligne], 5 | 2013, mis en ligne le 31 décembre 2013, consulté le 23 septembre 2020. URL : http://journals.openedition.org/lrf/984 ; DOI : https://doi.org/10.4000/Irf.984

Ce document a été généré automatiquement le 23 septembre 2020

(C) La Révolution française 


\title{
Le républicanisme anglais dans la France des Lumières et de la Révolution : mesure d'une présence
}

\author{
François Quastana et Pierre Serna
}

$1 \quad 1792$ est pour les Français le moment de naissance de la République dont on a célébré l'année dernière le $220^{\mathrm{e}}$ anniversaire. Mais si la République est née institutionnellement à cette date, cette dernière ne constitue pas, loin s'en faut, l'an zéro du républicanisme. Elle est le fruit d'une plus longue histoire ${ }^{1}$, d'évènements, d'expériences et d'idées qui ont contribué à la préparer et à la faire éclore ${ }^{2}$. C'est à l'étude d'une partie de sa genèse et de ses racines politiques, philosophiques et culturelles qu'est consacré ce nouveau numéro de La Révolution Française.

2 Alors que les travaux sur le républicanisme se sont multipliés ces dernières années outre-Manche et outre-Atlantique, dans la lignée des études fondatrices de Zera Fink ${ }^{3}$, Caroline Robbins ${ }^{4}$, John Pocock ${ }^{5}$, Quentin Skinner ${ }^{6}$, Philip Pettit ${ }^{7}$, l'apport de la pensée républicaine anglaise tel qu'il se manifeste à partir de la fin du XVII et du XVIII ${ }^{\mathrm{e}}$ siècle en France n'a pas fait véritablement l'objet d'une étude d'ensemble malgré l'appel lancé par Keith Michael Baker ${ }^{8}$, reçu et renouvelé récemment par Rachel Hammersley9.

Le retard, en voie d'être comblé, pris par les études dix-huitiémistes et révolutionnaires dans l'analyse de l'apport du langage du « républicanisme classique » et de l'expérience républicaine anglaise à l'imaginaire et à l'idéologie révolutionnaire française peut sans doute s'expliquer par la conviction profonde, longtemps ancrée dans la communauté scientifique, de l'existence d'une exception française ${ }^{10}$, un topos historiographique qui a longtemps paralysé toute tentative de compréhension plus large du phénomène révolutionnaire français en empêchant de le replacer dans la chaine de la Révolution atlantique ${ }^{11}$. Sans nier la singularité de chaque expérience républicaine, française ou étrangère, on peut estimer que ce mythe de l'exception a interdit de penser le premier moment républicain français comme l'actualisation ou la réinvention d'une tradition républicaine antérieure dont les origines remontent à l'expérience des Républiques italiennes de la Renaissance, aux écrits de Machiavel et ceux de ses disciples 
républicains anglais ou hollandais du XVII et $\mathrm{XVIII}^{\mathrm{e}}$ siècles. Cet héritage a été recueilli de façon non univoque par des auteurs de langue française comme Rousseau, Mably, Saige, Brissot ou Mirabeau. A cela s'est ajouté le succès indéniable en France du paradigme la Liberté des anciens et de la Liberté des modernes théorisé par Benjamin Constant au XIX ${ }^{\mathrm{e}}$ siècle pour combattre les idées de Rousseau et de Mably et leurs disciples Jacobins. Ce paradigme dont la valeur heuristique est aujourd'hui largement questionner n'en a pas moins été repris par toute une série d'interprètes, ce qui a empêché de saisir dans toute sa complexité la nature du référent antique sous la Révolution par l'assimilation simpliste toute référence aux Républiques de l'Antiquité à une conception ancienne et révolue de la politique et/ou à un pur ornement rhétorique. Cette vision réductrice qui tendait à considérer qu'il n'y avait uniquement deux façons antinomiques de penser la liberté a longtemps conduit à négliger une troisième conception de la liberté, antérieure au libéralisme : la liberté républicaine conçue comme un idéal de non domination dont les travaux de Quentin Skinner ont grandement contribué à la redécouverte.

4 Enfin, l'opposition originellement établie par Pocock dans le Moment Machiavélien entre le langage des droits naturels et celui de la vertu civique a également contribué à masquer la rencontre féconde qui s'est opérée très tôt dans les écrits de Milton ou de Sidney entre ces deux langages à tort présentés comme rivaux et incompatibles ${ }^{12}$. Ces écrous conceptuels étant, pour la plupart, levés ou en passe d'être levés, cela laisse espérer à brève échéance un progrès considérable dans l'étude des origines de la République en France.

5 Les contributeurs de ce numéro se proposent de relever un défi historiographiques en pensait ensemble et de façon non conflictuelle les histoires de l'Angleterre et de la France. La violence de la seconde Guerre de Cent Ans entre 1690 et 1815, la virulence des guerres révolutionnaires puis impériales, et l'anglophobie qui en a résulté dans le récit de l'histoire nationale, ont effacé ce lien, évident aux hommes du XVIIIe siècle, entre les deux traditions républicaines de part et d'autre du Channel. Ensuite, au début du XIXe siècle, la fascination constante des élites libérales françaises pour le modèle de la monarchie parlementaire anglaise n'a pas peu contribué à son tour à la construction de l'oubli des liens de ces deux écoles de républicains radicaux. Les auteurs d'article de ce numéro se proposent de retrouver les traces, les liens, les indices qui permettent de penser les connexions de cette famille républicaine disparue pour des raisons diplomatiques et idéologiques et retrouvée aujourd'hui en ce moment de recherche sur les origines du républicanisme ${ }^{13}$. Il a fallu, pour cela, mesurer à travers une période longue l'apport des idées et de l'expérience républicaines anglaises dans la formation d'une culture contestataire de l'absolutisme monarchique, qui a émergé dès la fin du règne de Louis XIV et s'est développée tout au long des règnes de ses successeurs avant d'atteindre son apogée sous la Révolution. Cet «esprit républicain», ce "républicanisme", ces expressions sont employées avec une connotation négative dans le vocabulaire politique du dernier siècle de l'Ancien Régime pour désigner une pensée séditieuse qui met l'accent sur la souveraineté populaire, la séparation des pouvoirs, la vertu civique et la participation nécessaire du peuple, sinon à l'exercice du pouvoir, du moins à son contrôle sont toujours associés à l'Angleterre que ce soit sous la plume de Bayle ${ }^{14}$ ou celle du Marquis d'Argenson ${ }^{15}$.

6 La genèse de la tradition républicaine anglaise est à rechercher dans les années 1642-1660 durant lesquelles éclatent la guerre civile et la première révolution qui 
s'achève avec le régicide de Charles $\mathrm{I}^{\mathrm{er}}$ et l'instauration du Commonwealth par Cromwell. Elle s'est étendue sur plusieurs générations jusqu'aux dernières décennies du XVIII siècle ${ }^{16}$.

7 La première génération des républicains anglais eut pour leaders John Milton, secrétaire d'Etat du Conseil d'Etat de la République et auteur d'importants textes politiques, Marchamont Nedham, directeur du Mercurius Politicus, organe de propagande du nouvel Etat et qui publia The Excellency of a free State en juin 1656 et surtout James Harrington qui fit paraître la même année, sa célèbre Oceana ${ }^{17}$.

8 L'héritage idéologique de ces lecteurs fervents des Discours sur la première décade de TiteLive de Machiavel fut recueilli et ranimé au début des années 1680 par des auteurs comme Algernon Sidney dans ses Discourses concerning government ou Henry Neville, l'éditeur des œuvres de Machiavel en langue anglaise, dans son Plato Redivivus, pour attaquer le papisme et les règnes «tyranniques » des derniers Stuarts, s'inscrivant en porte à faux avec les théories absolutistes de Thomas Hobbes et Robert Filmer développées en grande partie dans le but de réfuter la conception républicaine de la liberté ${ }^{18}$.

9 Ce legs républicain fut transmis aux générations suivantes par l'intermédiaire d'écrivains comme John Walter Moyle ou John Toland, joua un rôle essentiel dans la controverse autour de la "standing army " à partir de 1685 et en vint à former la composante principale de la pensée politique des Radicals et des Country Whigs. Ce radicalisme whig trouva l'une de ses expressions les plus caractéristiques au cours dans les années 1720 dans les célèbres Cato's Letters, sous la plume de John Trenchard et de Thomas Gordon, avant de faire l'objet d'une récupération opportuniste par Bolingbroke et les membres de son cercle dans leur critique de l'oligarchie Whig de Robert Walpole ${ }^{19}$. C'est dans ce même patrimoine idéologique que puise l'historienne Whig Catherine Macaulay pour réhabiliter l'expérience du Commonwealth comme la véritable époque de la liberté et saluer la déposition d'un roi tyrannique puis Richard Price et les colons américains pour justifier la Révolution américaine et la rupture avec une métropole accusée d'avoir corrompu la constitution anglaise, au nom d'une théorie néo-romaine de la libertée ${ }^{2}$.

La tradition républicaine et le langage qu'elle véhicule sur la liberté et l'esclavage, la vertu et la corruption, l'histoire romaine, la milice citoyenne et les armées permanentes, le régime mixte, le luxe et le commerce, le règne de la loi et le despotisme, l'origine populaire de la souveraineté, la lecture républicaine de Machiavel ont été reçus en France dès la fin du XVII siècle, avant d'influencer nombre de penseurs des Lumières dans leur critique de l'absolutisme pour constituer in fine un formidable arsenal d'idées et un incroyable réservoir de concepts pour les révolutionnaires français. En France, la réception de la tradition républicaine anglaise apparaît concomitante de l'essor d'une lecture républicaine des œuvres du Secrétaire de la République florentine, lecture, encouragée notamment par les écrits de Sidney ou de Gordon.

11 Si certains travaux sont venus, ces dernières années, éclairer certains aspects de cette réception ${ }^{21}$, de nombreux points aveugles demeurent dans l'historiographie moderniste et révolutionnaire concernant cette réception: A partir de quelle époque, les idées républicaines anglaises ont-elle commencé à pénétrer et essaimer en France ? Quels ont été les vecteurs de leur réception et de leur transmission? Qui sont les auteurs, les traducteurs qui ont joué le rôle de passeurs des textes canoniques du républicanisme 
anglais sur l'autre rive du chanel? Quelle était leur intention en faisant connaître ses écrits au public français? Quels sont les œuvres et les auteurs qui ont été privilégiés par les traducteurs? Quel a été l'impact de cette réception? Quelle part la République anglaise, comme matrice d'idées, dans la production écrite de deux histoires comparées, sous la forme de controverses théoriques, a-t-elle occupée dans la formation du républicanisme français? Autant d'interrogations auxquelles les auteurs des contributions réunies dans ce numéro de La Révolution française se sont proposés de fournir une première série de réponses. Ce numéro est en grande partie construit à partir des réflexions menées dans le cadre de journées d'études internationales organisées à la faculté de droit d'Aix-en-Provence les 25 et 26 mai 2012 par l'Institut d'Histoire de la Révolution Française de l'Université Paris 1 Panthéon-Sorbonne (UMS 622) et le Centre d'Etudes et de Recherches d'Histoire des Idées et des Institutions politiques (EA 2186) de l'Université d'Aix-Marseille (AMU).

Intitulée "Le républicanisme anglais dans la France des Lumières et la Révolution ", cette rencontre transdisciplinaire qui a réuni Historiens, Historiens du droit et Philosophes politiques, s'ordonnait autour deux principales problématiques: La réception des grands textes républicains anglais au XVIII ${ }^{\mathrm{e}}$ siècle, des dernières décennies du règne de Louis XIV aux Lumières tardives; et l'utilisation des références républicaines anglaises à l'époque révolutionnaire.

13 Toute réception implique nécessairement, on le sait, une transformation de l'objet reçu, conformément au vieil axiome scolastique médiéval: «Quidquid recipitur, ad modum recipientis recipitur». Les problématiques retenues renvoient à une difficulté méthodologique, classique en Histoire des idées politiques: celle de la réception du texte d'auteurs, qui plus est étrangers, par d'autres auteurs, c'est-à-dire de la lecture, de la compréhension et de l'utilisation de leurs thèses, dans un contexte étranger à leur élaboration.

C'est à ce type d'analyse qu'ont tenté respectivement de se livrer François Quastana et Eric Gasparini, à propos de la réception des œuvres de Sidney et de Gordon, en partant des premiers récepteurs, notamment des traducteurs et des premiers transmetteurs, des " passeurs ", ceux qui ont permis la première diffusion des idées contenues dans ces textes puis en envisageant les récepteurs secondaires, ceux qui portent témoignage de l'audience acquise par ces écrits au sein des Lumières, que ce soient d'ailleurs pour s'en approprier les idées ou pour les réfuter. Les réfutations, c'est le cas de celle du Marquis d'Argenson à propos des Discours sur le Gouvernement de Sidney, nous en apprennent parfois autant sur l'audience acquise par ces textes que bien des comptes-rendus élogieux. Ces deux contributions mettent en exergue le rôle majeur du Refuge Huguenot, à travers notamment les gazettes hollandaises et les traductions dans la diffusion des thèses républicaines en soulignant les liens ténus existant, à l'origine, entre républicanisme anglais et protestantisme français. Elles analysent ensuite comment à partir de cette réception originelle les écrits de Sidney et ceux de Gordon ont pu essaimer et connaitre un succès important auprès d'un public beaucoup plus large en devenant deux références fondamentales des philosophes de Barbeyrac à Rousseau en passant par Montesquieu, Voltaire, d'Holbach et Diderot, puis des révolutionnaires français.

15 A travers une relecture originale des œuvres de Montesquieu, Raymonde Monnier a montré comment, à partir des sources républicaines anglaises, l'aristocrate parlementaire a procédé dans l'Esprit des Lois, à une instrumentalisation du langage et 
des thèmes républicains au service de son projet de monarchie tempérée par les corps intermédiaires. Sa contribution éclaire d'un nouveau jour les liens ambigus qui unissent l'un des auteurs phares des Lumières françaises et le républicanisme anglais et la distance qui sépare sa conception de la liberté et de la conception républicaine recueillie et raffinée par Rousseau.

L'étude de la Révolution française est venue quant à elle, confirmer le rôle fondamental du référent républicain anglais dans l'idéologie révolutionnaire. On peut à ce sujet s'interroger, comme y invite Christopher Hamel, sur l'utilisation de l'expression "républicanisme classique " pour qualifier l'adaptation de "l'esprit républicain du XVII ${ }^{e}$ siècle anglais » à la France de la Révolution. Si l'on doit se garder d'assimiler de façon sommaire, comme ont pu parfois paraître le faire Baker et d'autres commentateurs, le « républicanisme classique " à la «Liberté des anciens » et l'opposer systématiquement au républicanisme jusnaturaliste moderne, il n'y a en revanche désormais plus lieu de douter de sa présence. C'est ce que confirme l'étude de Pierre Serna du Patriote français de Brissot entre 1789 et 1791 qui souligne la «place discrète mais tenace » du républicanisme anglais dans le journal du girondin anglomane et ami des Noirs. C'est un double visage de l'Angleterre qui s'offre aux lecteurs sous la plume de Brissot, matrice originelle du républicanisme à travers ses grands auteurs, Milton Sidney, Locke ou Macaulay mais dont les épisodes révolutionnaires témoignent de l'impuissance à engendrer une république durable. C'est ce qu'ont réalisé en revanche les colons américains, fils rebelles de mère-patrie britannique et principaux légataires de l'héritage de l'idéologie et des principes du Commonwealth à la fin du XVIII siècle dont le pragmatisme de l'expérience doit, selon Brissot, servir d'exemple à la France.

La présence obsédante du référent historique et républicain anglais se lit également dans les débats autour du procès du Roi à la Convention à la fin de l'année 1792 analysés par Clizia Magoni. La comparaison du procès de Louis Capet avec celui de Charles Stuart s'impose comme une évidence aux yeux des contemporains tant chez les partisans que chez les adversaires de la monarchie. Dans ce débat crucial, deux œuvres sont principalement convoquées par ceux qui souhaitent justifier la mort de Louis XVI : la Pro populo anglicano Defensio de Milton traduite en français dès 1789 par Jean-Baptiste Salaville et publiée sous l'illustre patronage de Mirabeau et l'Histoire d'Angleterre depuis l'avènement de Jacques Ir jusqu'à la Révolution de Catherine Macaulay Graham, également traduite en français en partie par le tribun aixois mais publiée après sa mort par Guiraudet. En réalité Mirabeau visait un autre objectif. Il travaillait à l'établissement d'une monarchie régénérée aux sources du patriotisme et au renforcement du pouvoir exécutif. Pourtant les textes possédaient par eux même un potentiel subversif qui la conjoncture de la Révolution française et sa radicalisation allaient révéler.

Les enjeux politiques de la traduction de l'Histoire de la « Porcia de l'Angleterre » sont dévoilés avec précision par Francesco Dendena qui montre l'importance de cette œuvre en tant qu'outil intellectuel pour penser la rupture révolutionnaire et comme grille de lecture de l'histoire de la Révolution en train de se faire au miroir des révolutions anglaises. Pour Brissot comme pour Mirabeau, fervents lecteurs de l'historienne dès les années 1780 , son œuvre à l'instar de celles de Machiavel, Sidney ou Gordon permet de penser la problématique de la liberté politique en termes de conflit et de lutte. La liberté est une conquête du peuple, la Révolution américaine qui est regardée par Mirabeau et Brissot comme un prolongement de la lutte séculaire des Whigs contre la tyrannie l'illustre parfaitement. A l'automne 1791, néanmoins, sous la plume de 
Guiraudet dont la préface du troisième volume de la traduction résonne comme un avertissement contre le risque d'emballement dangereux du processus révolutionnaire, les leçons de l'histoire républicaine anglaise se muent en «plaidoyer en faveur de la monarchie des Bourbons".

Après la chute de la royauté, l'auteur républicain anglais qui paraît avoir bénéficié de l'audience la plus forte auprès des révolutionnaires semble sans conteste Harrington, dont le républicanisme, pourtant souvent qualifié d' "aristocratique » et d'" élitiste " apparaît déjà porteur, selon Rachel Hammersley, de l'idéal démocratique moderne qui s'empare de la France à l'été 1792. Défendu en Angleterre par les Niveleurs, le terme démocratie prend sous la plume d'Harrington et des membres de son cercle une connotation positive qui contraste singulièrement avec l'acception traditionnelle du terme depuis Aristote et dans la pensée républicaine anglaise du XVII ${ }^{e}$ siècle en général. Déjà recueillis par Rutledge dès 1785 , les ferments démocratiques de la pensée harringtonienne se diffusent par l'intermédiaire de Sieyès avant de constituer l'une des principales sources d'inspiration de Camille Desmoulins et d'autres membres du Club des Cordeliers comme Louis de la Vicomterie, Théophile Mandar et Théodore Lesueur. L'Oceana devient un bréviaire de l'art du législateur pour penser l'instauration d'un système démocratique adapté à l'étendue des grands Etats modernes et la subtile alchimie entre les lois et les mœurs.

20 Après la chute de Robespierre, souvent peint sous les traits de Cromwell et de Catilina, la référence républicaine anglaise connaît un regain d'actualité sous le Directoire et jusqu'au lendemain du 18 Brumaire.

21 L'automne de la Révolution, étudié par Daniele Di Bartolomeo, illustre la permanence des références à l'expérience républicaine anglaise avec la traduction française en 1795 des œuvres politiques d'Harrington et la republication des œuvres de Sidney et Gordon. La comparaison des deux révolutions s'impose aux contemporains, non seulement pour en étudier les causes mais pour tenter de deviner à travers l'histoire de la première Révolution anglaise le devenir de la française. L'influence anglaise se mesure également dans l'adhésion des Thermidoriens au vieil idéal de la constitution mixte revisitée par les républicains américains à travers le système de l'équilibre des pouvoirs sensé prévenir la dégénérescence inéluctable de la République et de la liberté et la chute du pouvoir dans les mains d'un nouveau Cromwell ou César. Une crainte et une comparaison que s'efforcera de repousser Bonaparte après son accession au pouvoir, mettant en exergue la spécificité de la situation française.

La Revue électronique « La Révolution française » grandit et propose à ses lecteurs trois nouvelles rubriques. La première, "Varia ", sera consacrée à un article de recherches. Antonio de Francesco inaugure cette rubrique avec un article érudit et inédit sur la position d'Alphonse Aulard, face à la Révolution russe. La seconde verra un long compte rendu de lecture. Plutôt que de multiplier les rubriques de livres mentionnés, il a été décidé de solliciter les jeunes chercheurs et de leur demander des analyses conséquentes, soit sur des livres d'actualité, soit sur des livres qui seraient passés inaperçus des historiens du champ révolutionnaire. C'est le cas de Mathieu Féradou lisant le texte de David V. Erdman, Commerce des Lumières. John Oswald and the British in Paris, 1790-1793, paru en 1986, et toujours fort utile. Enfin une Rubrique « la Révolution, aujourd'hui » constitue une tribune plus libre qui désire rendre compte de l'actualité de l'histoire de la Révolution et de son utilisation dans les espaces politique, culturel et médiatique actuels. Dans cette perspective, un retour sur la récente controverse à 
propos de la reconstitution du visage de Robespierre s'imposait, avec un regard décalé et non soulevé jusque-là sur la masculinité du visage proposé.

\section{NOTES}

1. Eric Gojosso, Le concept de République en France XVI ${ }^{e}$-XVIII ${ }^{e}$ siècle, Aix-en Provence, PUAM, 1998.

2. Michel BIARD, Philippe BOURDIN, Hervé LEUWERS, Pierre SERNA (dir.), 1792. Entrer en République, Paris, Armand Colin, 2013.

3. Zera S. FINK, The Classical Republicans: An Essay on the Recovery of a Pattern of Thought in Seventeenth-Century England, Northwestern University Press, 1945.

4. Caroline A. RoBbins, The Eighteenth-Century Commonwealthman, Harvard University Press, 1959.

5. John G. A. Pососк, The Machiavellian Moment: Florentine Political Thought and the Atlantic Republican Tradition, Princeton University Press, 1975.

6. Quentin SKINNER, La liberté avant le libéralisme, Paris, Seuil 2000; Quentin SKINNER, "Classical Liberty, Renaissance Translation and the English civil War ", dans Quentin SKINNER, Visions of Politics, Cambridge, Cambridge University Press, 2002, vol. II, p. 308-343

7. Philip PETTIT, Républicanism, A Theory of Freedom and Government, New York, Oxford University Press, 1997.

8. Keith BAKER, «Transformations of classical Republicanism in Eighteenth-Century France ", The Journal of Modern History, 2001, vol. 73, n 1, p. 32-53.

9. Rachel HAMMERSLEY, The English Republican Tradition and Eighteenth-Century France: Between the Ancients and the Moderns, Manchester, Manchester University Press, 2010.

10. Michel vovelle (dir.), Révolution et République. L'exception française, Paris, Editions Kimé, 1994.

11. Annie JOURDAN, La Révolution, une exception française?, Paris, Flammarion, 2004 ; Pierre SERNA (dir.), Républiques scurs. Le Directoire et la Révolution atlantique, Rennes, Presses Universitaires de Rennes, 2009 ; Manuela ALBERTONE, Antonino DE FRANCESCo (dir.), Rethinking Atlantic Word. Europe and America in the Age of Democratic Revolutions, New York, Palgrave Macmillan, 2009.

12. Voir notamment Christopher HAMEL, L'Esprit républicain. Droits naturels et vertus civique chez Algernon Sidney, Paris, Classiques Garnier, 2011.

13. Voir cependant les travaux pionniers d'olivier LUTAUD, «Des Révolutions d'Angleterre à la Révolution Française : l'exemple de la liberté de la presse ou comment Milton ouvrit les Etats généraux ", dans Jean-Claude BONNET et Philippe ROGER (dir.), La Légende de la Révolution, Clermont Ferrand, 1988, p. 115-125; Olivier LUTAUD, «Emprunts de la Révolution française à la première révolution anglaise », RHMC, 37, 1990, p. 589-607.

14. Pierre BAYLE critique "l'esprit républicain» des Refugiés Huguenots (Avis important aux réfugiés sur leur prochain retour en France. Donné pour estrennes à l'un d'eux en 1690. Par monsieur C.L.A.A.P.D.P, Amsterdam, Jaques Le Censeur, 1690, p. 5-6).

15. Le néologisme "républicanisme » est employé par d'Argenson dès 1756 (Mémoires et journal inédit du Marquis d'Argenson, Paris, Janet, 1858, t. V., p. 346). L'une des premières apparitions du terme se trouve chez Pierre Louis Saintard qui évoque à propos de Saint Domingue «les imputations odieuses de républicanisme" dont on accuse les colonies. (Essai sur les colonies françoises, sl., 1754, p. 126). On trouve également mention du terme dans une lettre anonyme adressée à Louis XIV qui signale la tendance au "républicanisme» des philosophes, des 
protestants et des jansénistes (Quintin CRAUFURD, Mémoires de Madame du Hausset, femme de Chambre de Madame de Pompadour, Paris, Baudoin, 1824, p. 133).

16. Blair WORDEN, The English Civil Wars 1640-1660, Londres, Weidenfeld \& Nicolson, 2009.

17. Jonathan sсотт, Commonwealth Principles. Republican Writing of the English Revolution, Cambridge, Cambridge University Press, 2004.

18. Quentin SKINNER, Hobbes et la conception républicaine de la liberté, Paris, Albin Michel, Bibliothèque des idées, 2009.

19. Isaac KRAMNICK, Bolingbroke and his circle: The Politics of Nostalgia in the Age of Walpole, Cornell University Press, 1992, (1968), p. 243 et suivantes.

20. Quentin SKINNER, La liberté avant le libéralisme..., op. cit.

21. Voir Johnson Kent WRIGHT, A Classical Republican in Eighteenth-Century France: the Political Thought of Mably, Stanford University Press, 1997 ; Jean Fabien SPITZ, La liberté politique : Essai de généalogie conceptuelle, Paris, PUF, 1995 ; Jean Fabien SPITZ, L'amour de l'égalité. Essai sur la critique de l'égalitarisme républicain en France 1770-1830, Paris, Vrin-Éditions de l'EHESS, « Contextes », 2000 ; Edouard TILLET, La constitution anglaise. Un modèle politique et institutionnel dans la France des Lumières, Paris, PUAM, 2001; Andrew JAINCHILL, "The Constitution of the Year III and the Persistence of Classical Republicanism», French Historical Studies, Vol 26, $\mathrm{n}^{\circ} 3$, 2003,

p. 399-435; Raymonde MONNIER, Républicanisme, Patriotisme et Révolution française, Paris, L'Harmattan, 2005 ; Raymonde MONNIER, «Traduction, transmission et Révolution: Enjeux rhétoriques de la traduction des textes de la conception républicaine de la liberté autour de 1789, AHRF, 2011/4, n³64; François QUASTANA, La pensée politique de Mirabeau (1771-1789): "Républicanisme classique " et régénération de la monarchie, Aix-en-Provence, PUAM, 2007 ; Marc BELLISSA, Yannick BOSC, Florence GAUTHIER, Collectif L'Esprit des Lumières et la Révolution, Républicanismes et droit naturel. Des humanistes aux révolutions des droits de l'homme et du citoyen, Paris, Editions Kimé, 2009. 